\title{
Cultural Diversity in the Practice of Physical Therapy: A Review of the Literature
}

\author{
James McKivigan \\ School of Physical Therapy, Touro University Nevada, Henderson, Nevada, USA \\ *Corresponding Author: James McKivigan, School of Physical Therapy, Touro University Nevada, \\ Henderson, Nevada, USA
}

\begin{abstract}
:
Background:

Cultural competence is a term that encompasses one's capacity for understanding, communication, and effective interactions with individuals across the cultural spectrum. Recognition of these principles and practical training focused on relevant skills and abilities are needed to prevent discriminatory practices based on patient age, ethnicity, socioeconomic status, race, religion, gender identification, and sexual orientation. A culturally competent physical therapist will be able to use these skills and abilities to promote effective communication with all patients and remove barriers to obtaining appropriate health care.
\end{abstract}

\section{Methods:}

Toward this end, this study includes a review of the recent literature and focuses on interventions used to promote cultural diversity and cultural competence in the practice of physical therapy.

\section{Conclusion:}

Although the study designs and outcomes were varied and complex, the findings highlight several validated tools that can be used to evaluate the impact of critical interventions. Future primary studies and reviews must be explicit concerning their working definition of cultural competency and might focus on quantitative evaluations of physical therapy students and practitioners' interventions.

Keywords: Cultural competency, medical education, health services research, physical therapists, review

\section{INTRODUCTION}

The physical therapy profession must be committed to improving cultural competence to effect societal transformation in education, practice, and research. All future health care providers will need to consider cultural competence as a significant factor when providing patient care. The holistic basis of cultural competence includes acquiring a critical set of skills, professional policies, attitudes, and behaviors that facilitate patient care in cross-cultural settings. It is essential to recognize that factors that shape cultural competence are not restricted to ethnicity or race. Indeed, cultural competence also involves understanding the impact of religion, age, language, socioeconomic status, sexual orientation, and gender identification when considering patient responses in a given health care setting. Training for cultural competence focuses on the understanding and the appreciation of individual differences and behaviors and the identification of biases that may influence the nature and scope of care provided to individual patients.

The ability to attain high levels of cultural competence will require ongoing effort and interest in this goal. Toward this end, the Office of Minority Health of the Agency for Healthcare Research and Quality of the U. S. Department of Health and Human Services has recently emphasized the need for culturally competent health care. Concepts underlying cultural competence in physical therapy focus on the understanding of how attitudes and behaviors become integrated into cultural issues and how this information might be used to deploy new policies, guidelines, and activities for professional development[1]. Physical therapists need to have a broad understanding of the factors associated with cultural competence to appreciate the impact of socioeconomic and family status on patients' 
decisions. Indeed, all medical professionals would benefit from a clear understanding of the factors contributing to individual choices regarding health and health care.

Cultural competence is essential to prevent discriminatory practices based on patient age, ethnicity, socioeconomic status, race, gender, sexual orientation, and religion.[2] For example, many significant health issues are directly associated with socioeconomic issues. This fact will need to be appreciated by all physical therapists who seek to communicate effectively with patients. An effective physical therapist is one who conveys information precisely and succinctly in layman terms that can be understood by all patients, regardless of their level of education.

A culturally competent physical therapist can appreciate cross-cultural relationships and can use this knowledge when providing health care to patients. Racial and ethnic minority groups in the U.S. experience significant differences in the quality of healthcare that they receive. A culturally competent physical therapist will understand barriers to patient access and promote effective communication. Likewise, language barriers create problems that are recognized worldwide. Mutual respect must be the basis of any interactions between the physical therapist and the patient. This point is critical, as many patients believe that health care providers do not have a clear understanding of their needs.[3] Many patients report that they do not trust healthcare providers due to past stereotyping and discriminatory behaviors. Feelings of mistrust will serve as roadblocks to any efforts to create meaningful relationships with patients.

The socioeconomic impact of all decisions must be considered, as patients will be evaluating the costs involved when considering physical therapy. Many uninsured or under-insured patients will be unable to pay for treatment, and those covered by government programs such as Medicaid or Medicare may be denied specific services. A culturally competent physical therapist should understand the differences in perspective that emerge from this paradigm and patient perceptions regarding self-care. Notably, patients may have different perceptions about which therapies are essential for their health and well-being.[4] The culturally competent physical therapist should also have a clear understanding of how to explain the nature and impact of complex therapies to non-professionals. This review of a series of published review articles that address cultural competence and cultural diversity among physical therapists follows the guidelines provided by the Preferred Reporting Items for Systematic Reviews and Meta-Analyses (PRISMA) statement.

\section{METHODS}

\subsection{Eligibility Criteria}

This review included results evaluated in reviews of controlled and non-controlled intervention studies published in peer-reviewed journals only. Case reports, retrospective studies, expert opinions, case series, and letters to the editors were not included in this review. Studies were limited to those in English. Eligible studies noted the intent to improve and evaluate cultural competence and cultural diversity among physical therapists. Studies at centers that maintained high cultural diversity levels among health care providers were not included in this study.

\subsection{Search Strategy}

A complete search of the literature was performed, including publications listed in MANTiS, Scopus, and CINAHL databases from January 2000 to June 2012. The Cochrane Library was used to identify reviews that focused on cultural competence or cultural diversity in all health care settings. Synonyms, alternative terms, and spelling and versions of both "intervention" and "condition" were used. Bibliographies and relevant review articles were searched by hand to identify additional eligible studies.

\subsection{Study Selection}

Citations from the initial electronic searches were integrated into a single database, and duplicate records were eliminated. Two reviewers considered all titles and abstracts and eliminated sources that were not relevant to this study. The two independent reviewers evaluated full-text versions of selected publications to assess eligibility for inclusion, and any differences of opinion were resolved by consensus involving a third independent reviewer. 


\subsection{Exclusion and Inclusion Criteria}

Reviews that met the initial eligibility criteria were those that were written in English and included:

Qualitative, quantitative, or mixed methods.

Participation of health care administrators, patients, and health care practitioners and clinicians, patients, or other users of health care services.

A determination of the cultural diversity in a given physical therapy practice and interventions intended to enhance cultural competency.

Interventions at community health services, hospitals, and educational institutions.

Evaluations of outcomes at the system, organizational, or individual level.

Reviews that were excluded include those that (1) determined cultural competence in non-healthcare settings before 2009 or (2) did not have a Methods section.

\subsection{Quality of Review}

Information from Health Evidence ${ }^{\mathrm{TM}}$ includes five questions that can evaluate the quality of individual review articles with evidence-informed techniques. Among these points, each review of primary findings should be performed by two authors. This tool was used to generate strong, moderate, or weak assessment ratings for each of the review articles considered here.

\section{RESUltS}

\subsection{Identification}

A total of 6660 manuscripts were identified in the initial database search. The abstracts and titles were examined for eligibility using the inclusion and exclusion criteria noted above. To minimize the potential for bias, only data related to the inclusion criteria were extracted from each of these reviews. Most of the studies that emerged from this evaluation were published between 2007 and 2012 ( $\mathrm{n}=$ 15). The review articles selected explicitly focused on health care settings and included findings based on analyses of health care systems and health care professionals. The studies included pre-study and post-study designs, randomized controlled trials, and qualitative analyses. Many of the reviews considered the concept of cultural competency and offered individual evaluations of specific outcomes. For example, Smith et al. (2001) reviewed two meta-analyses that satisfied the inclusion criteria noted here. Using practical appraisal tools such as those provided by the Centre for EvidenceBased Medicine (Oxford, UK), the quality of 15 reviews was considered.

\subsection{Interventions to Enhance Cultural Competency}

All review articles eligible for inclusion in this study included interventions aimed at enhancing cultural competency and diversity. These interventions included workshops for health care practitioners, patient-based programs, patient navigators, and peer education programs.

Most of the reviews included in this study included interventions designed to improve healthcare providers' and physical therapists' cultural competencies. Only a few reviews included cultural competency interventions that were focused, specifically on patients. Some studies examined cultural competency at the organizational level and addressed factors associated with acquiring culturally sensitive health care programs for patients.

Only one review identified a specific intervention focused on promoting culturally competent practices for organizations and health care personnel. Bhui et al. (2007) examined the cultural competence models in mental health.

\subsection{Study Outcomes}

The three primary objectives of the reviews considered in this study were assessments of patientrelated outcomes, health care provider-related outcomes, and outcomes associated with access to health services. Models of cultural competency models were also evaluated. 


\subsection{Provider Outcomes}

Health care providers' objectives focused on results reflecting attitudes, skills, and knowledge of factors associated with cultural competency. Beach et al. (2005) reviewed patient-provider outcomes with concepts, including traditional cultural practices[5]. These studies measured outcomes related to the concept of attitude, which included the cultural basis of self-efficacy and provider interest in learning about patients and their family backgrounds. Skills involving communication and the use of treatment plans were also implemented in the health care process. Smith et al. (2001) considered multicultural competencies a significant outcome measure, while Kokko (2011) evaluated nursing students' cultural knowledge.

\subsection{Patient Outcomes}

Patients and patient-associated evaluations included physiological outcomes such as blood pressure, blood glucose, and body weight, and also focused on patient trust and satisfaction with the overall experience. Behavioral outcomes, including exercise and dietary preferences, were also evaluated in these reviews. Patient-focused interventions were used to enhance participation in the treatment of numerous disease processes.

\subsection{Health Service Access Outcomes}

Outcomes associated with access to health care services include interventions that involve patient navigators, interpreters, and community health care workers. These interventions were developed to furnish health care workers with the means to provide individualized care as part of the organizational culture.

Several of these reviews focused on the cost-effectiveness of these interventions. Beach et al. (2005) found that only 3 of the 25 studies provided information on cultural competence training[5]. The study published by Marin et al. (2017) evaluated the cost-effectiveness of training and provided a rough estimate of the overall costs; this limited focus studies the number of studies that were assessed in this review. An initial evaluation of all data included in the final set of review articles was performed. Due to overall heterogeneity, a meta-analysis could not be conducted.

\section{DISCUSSION}

\subsection{Ultimate Review Size}

This review considered articles published between January 2000 and June 2012 have identified specific problems and limitations when considering current interventions designed to enhance cultural competency in health care. Comparisons among these reviews revealed substantial heterogeneity concerning the intervention used, the specific health care providers and health care settings involved, characteristics of the patient populations, and health care outcomes considered. These results suggest that the translation of these findings to research and practice settings may involve significant complexities. However, most reviews report that cultural competency training has positive effects on provider outcomes. As such, these findings suggest that this type of intervention may be useful for promoting change in the health care setting.

The reviews included in this study were used to compare the outcomes of various types of intervention strategies. The most significant results were those obtained from studies focused on culturally trained health care workers and physical therapists. Many primary studies included designs focused on other interventions as well.

The reviews included in this study were also highly complex, limiting the ability to compare the various frameworks associated with cultural competency outcomes. The lack of uniformity might influence the acceptance of one or more of the strategies used to measure results; this problem might also prevent consensus required to initiate cultural competency interventions at other institutions. Future work will need to generate agreements on definitions and terminologies used to examine evidence from cultural competency interventions.

Bialocerkowski et al. (2011) reported that there is some potential for improvement in schools that teach physical therapy in culturally diverse academic environments. They also note a significant demand for resolving cultural diversity issues, specifically in this setting. 
These researchers reported positive perceptions of the attention given to efforts to accommodate cultural diversity. Their results imply that the field could benefit from studies that target recognition of cultural diversity via specific processes adopted by health care staff.

Cushman et al. (2015) focused on the impact of a workshop designed to teach cultural competency and evaluated outcomes from 25 participants enrolled in a Master of Public Health program. All the participants in this study agreed that this intervention resulted in improved self-awareness, although further improvements specifically designed to help students prepare for professional environments were recommended.

Dhaliwal et al. (2013) designed a study that analyzed an academic institution's environment with a specific focus on diversity, cultural competency, and inclusiveness. The researchers utilized previous work on this subject and found that diversity enhanced the learning environment, improved educational outcomes, and prepared the participants to work with diverse patient populations. This research study also revealed that increasing diversity in medical schools resulted in improved communication and creative problem solving and increased physicians' willingness to work in underserved areas. Furthermore, evaluating the outcomes of a training workshop focused on cultural education revealed that it facilitated changes in behaviors and perspectives relevant to cultural diversity. These conclusions were compatible with those reported previously and supported the concept of interactive workshops as an effective means to impart behaviors associated with cultural competency.

Esterhuizen and Kirkpatrick (2015) explored the relevant literature and theory while focusing on modern demands for cultural competency in professional work environments. Among the concepts considered, these researchers noted that many nations in the developed world are likely to experience significant diversity increases. There will no longer be any single (or majority) ethnic minority. The U. S. may reach this point as soon as 2050. As such, these researchers discussed calls for health care reforms that address the needs of numerous and diverse multicultural patient groups.

Hammerich (2014) offered a critical commentary of reports that focused on developing training programs designed to address cultural competency. The studies included in this commentary evaluated frameworks designed to integrate strategies used to improve cultural competency and provided recommendations on optimizing cultural competence through educational programs for chiropractors. The author concluded that chiropractic schools should review their plans and establish methods for prioritizing cultural competence training. This study used the theoretical framework from the Intercultural Development Continuum and focused on the potential to develop mindsets capable of accommodating a range of cultures. Interestingly, the results revealed that $98 \%$ of the participants over-assessed their cultural competency. Interestingly, participants who identified with minority groups were observed as among the more culturally competent. Overall, the study concluded that the instrument used for this evaluation was useful in assessing cultural competency.

Marin et al. (2012) reported that communication and transcultural skills were already integrated into physical therapy training and related medical education programs at one prominent university. The researchers found that both physiotherapy and medical education programs emphasized transcultural skills, although these skills were underemphasized in the dental training program. The researchers concluded that improvements were required and that a curriculum review process might address this concern. Furthermore, the researchers found that, while there were no statistically significant demographic differences across age or gender, there were significant differences between ethnicities when focused on results from evaluations of clinical reasoning, social efforts, and treatment, with minorities achieving overall lower scores in their final clinical placements.

Nuciforo (2015) conducted a study that assessed the current state of underrepresented minorities applying for positions in physical therapy training programs and considered variations in application trends compared to their Caucasian counterparts. The study noted that the statistical framework indicated that minorities accounted for only $20 \%$ of training program applications, although these groups represented a higher percentage of the U.S. population. The researcher found that Caucasians had overall higher undergraduate grade point averages and Graduate Record Examination scores than African Americans and Hispanics in the U.S. applicant pool. However, there were no substantial variations in the mean number of programs to which the different ethnicities applied. The study also revealed that undergraduate science grades were the primary predictor of admission for all years; none 
of the other factors evaluated achieved statistical significance. These findings were comparable to those published previously that focused on predictors of success at various stages. Overall, this study identified variables that might be used to predict admission to U.S. professional education programs for physical therapy.

Oliveira et al. (2015) aimed to develop a model that integrated interprofessional education into health care education programs. The researchers used existing frameworks to create and establish healthcare education programs with decentralized structures that required fewer resources than were needed by existing centralized systems. The researchers reported that the participants' increased depth of responses matched the scaffolding emphases of the specific learning programs. Furthermore, they concluded that organizations attempting to establish culturally inclusive educational objectives might benefit from a program that included assessing the cultural dimensions, developing a decentralized model, and examining the integrated roles of health and culture.

Palombaro et al. (2015) focused on potential increases in cultural competency following participation in directed education as a component of a physical therapy curriculum and determining whether additional clinical hours or pro bono clinical leadership facilitated cultural competency improvements. These researchers found that educational experiences in multicultural situations improved cultural competency in the student population under study. Students who volunteered for assignments involving culturally diverse cases were most likely to develop improved cultural competency. The researchers evaluated the relationships between general cultural competence perceptions, gained knowledge, capacity for reflection, and specific behaviors during consultations for cultural competency. The researchers also discussed how these evaluations could facilitate cultural competence improvements among students participating in training programs. Interestingly, the researchers found that all respondents scored low on general relevant knowledge and interpretation capacities but scored high on measures associated with reflection.

Villarruel et al. (2015) focused on the benefits of diversity in nursing environments, based on the argument that a diverse workforce will positively impact the health of the entire population. The researchers noted the need for change in this regard, as the nursing workforce did not accurately represent the U.S. population's diversity at large. They indicated that appropriate representation of all minority groups would be required to meet patient needs for communication and overall health. The researchers called for removing barriers to diversity and suggested that each employee might have the capacity to promote progressive change toward greater workforce diversity.

Wehbe-Alaman and Fry (2014) created a framework designed to establish and preserve an academic environment culturally sensitively, and that addressed the needs of a culturally diverse student body. They applied this framework to assess a group of Muslim students enrolled in the Doctor of Physical Therapy program at the University of Michigan. The researchers found that staff respondents were familiar with many aspects of Islam and faith-based cultural practices. The students recommended that there might be a greater emphasis on Islamic practices as initial components of the program curriculum. The study also examined the perspectives and experiences of physical therapists concerning ethnic diversity. The researchers found that, despite an apparent increase in variety, most therapists lacked experience working with minority groups. The researchers concluded that these therapists might not have the opportunity to develop the skills needed to address patient concerns (Yoswell, 2013). After considering the findings of other studies, the researchers stated that, although current observations reveal an increase in ethnic diversity in the profession, the general perception is that physical therapy remains a Caucasian-dominated career path.

Zanetti et al. (2014) assessed the impact of a culturally diverse inter-clerkship on participants' perspectives of information, their capacity to conduct interviews, and their empathy with culturally diverse student populations. This study also assessed the impact of student demographics on the learning process. The researchers found that increased exposure to cross-cultural communication facilitated improvements in knowledge, attitudes, and skills associated with cultural diversity.

Finally, Lu et al. (2012) evaluated various interventions' efficiency, including study settings, type of intervention, ethnic populations, and program delivery techniques. A clear understanding of the impact of a given intervention promoted interest and participation. However, the results focused on effectiveness were heterogeneous and somewhat complex when used to assess the effect on a specific patient or patient group. 


\subsection{Organizational Context}

Global views shape cross-cultural interactions. A given organization's culture can be generated by the physical setting, policy frameworks, and administrative arrangements. Interventions that help to enhance cultural competency might evaluate the organizational and well as the individual contexts. Training programs should be tailored to specific groups. For example, physicians would require particular skills and knowledge of the clinical tasks to succeed in a cultural competency program.

Cultural competency training has been an effective strategy that can enhance patient outcomes without dramatic organizational changes. The integration of cultural competency in corporate policy documents, including strategic plans and position statements, might be necessary to facilitate sustained growth. Likewise, there is evidence indicating a direct relationship between an organization's cultural diversity and the cultural diversity of its health care practitioners. The commitment and actions taken within the organization are effective at influencing the health care providers. The results of this study revealed that providers who were motivated to learn were most likely to be those who were already working in clinics with a culturally diverse staff.

\subsection{Self-Assessment Tools}

Although self-assessment is a practical approach for determining cultural competency, these methods include several subjective measures that may involve significant biases. These assessment measures included survey tools that include self-administered organizational checklists, provider selfassessment, and patient satisfaction. The overall efficacy of these tools has yet to be determined. At the individual level, self-rating might have an impact on cultural awareness. Systematic and organizational approaches are needed to assess cultural competency, including objective measures such as document review. Self-assessment is a significant step toward generating convincing evidence supporting the need to utilize cultural competency skills to enhance health outcomes for all patients. These research tools must be structured to evaluate organizational and individual level guidelines for cultural competency and how effectively each adheres to the delivery of service in the form of patient care.

It is not yet clear whether cultural competency can be attained without considering problems associated with white privilege and institutional racism. Discrimination and bias were mentioned in each of the reviews. Beach et al. (2005) reviewed these concepts as a component of the educational content. Beyond cultural differences, persistent racism will create even larger health disparities among patient groups. Self-awareness and personal reflection on one's individual and professional culture are both essential aspects of cultural competency.

Very few of the reviews considered here included evaluations of provider outcomes. These selfreflexive aspects are considered critical to any measure of cultural competency. Cultural awareness is not enough to combat issues associated with racism and its impact on health care disparities. Current training has been criticized for its focus on racial identities. Anti-racism training may provide meaningful contributions to cultural awareness and may influence society while avoiding adverse emotional reactions associated with "white guilt." Health care tends to manage culture as a factor associated with ethnicity and race, without reference to the economic, political, and social context. Suppose attention is limited to factors related to identity and culture alone. In that case, this might ultimately limit the use of practical techniques and may include a cultural focus that would foster stereotyping. Care should be taken to avoid overshadowing of culture when making clinical decisions. These cultural differences may result in discrimination issues. Factors such as poverty and limited education undoubtedly play a role in defining specific health care outcomes for various groups in the community. The risk factors associated with discrimination are among the essential aspects underlying socioeconomic disparities.

\section{CONClusions}

The intent of this review concentrated on methods that might be used to enhance cultural competency in health care settings. Toward this end, recent studies that address the evidence underlying current improvements and that assess prospects for future research have been evaluated. Most reviews focused on access to health care and advances in healthcare providers' outcomes and resource utilization. There was limited to no data available that addressed improvements based on interventions provided to patients or patients. 
This review focused on the complexities of research on cultural competency and identified areas of significant concern. These studies provided a uniform definition of cultural competence concerning health care that has been accepted in most countries. While there are many possible outcomes associated with direct interventions, several validated tools are currently used to determine cultural competency. Future primary studies and reviews must be explicit for the definition of cultural competency with a view towards what might be achieved by physical therapy practitioners; additional validated tools and models for determining cultural competency are needed. Multi-level interventions will be required to facilitate evaluations in more extended contexts, including those directed at the community and government policy. Taken together, these efforts will have an important impact on interventions designed to enhance cultural competency.

\section{REFERENCES}

[1] Bhui, K., Warfa, N., Edonya, P., McKenzie, K., and Bhugra, D. (2007). Cultural competence in mental health care: a review of model evaluations. BMC Health Services Research, 14: 15. doi: 10.1186/14726963-7-15.

[2] Anderson, L. M., Scrimshaw, S. C., Fullilove, M. T., Fielding, J. E., and Normand, J. (2003). Culturally competent healthcare systems. A systematic review. American Journal of Preventative Medicine, 14(3S): $68-79$.

[3] Whittemore, R. (2007). Culturally competent interventions for Hispanic adults with type 2 diabetes: a systematic review. Journal of Transcultural Nursing, 14(2): 157-66.

[4] Henderson, S., Kendell, E., and See, L. (2011). The effectiveness of culturally appropriate interventions to manage or prevent chronic disease in culturally and linguistically diverse communities: a systematic review. Health and Social Care in the Community, 14(3): 225-49.

[5] Beach, M. C., Price, E. G., Gary, T. L., Robinson, K., Gozu, A., Palacio, A., Smarth, C., Jenckes, M., Feuerstein, C., Bass, E. B., Powe, N. R., and Cooper, L. A. (2005). Cultural competence: a systematic review of health care provider educational interventions. Medical Care, 43(4): 356-73.

[6] Pearson, A., Srivastava, R., Craig, D., Tucker, D., Grinspun, D., Bajnok, I., Griffin, P., Long, L., Porritt, K., Han, T., and Gi, A. A. (2007). Systematic review on embracing cultural diversity for developing and sustaining a healthy work environment in healthcare. International Journal of Evidence-Based Healthcare, 14: 54-91.

[7] Fisher, T. L., Burnet, D. L., Huang, E. S., Chin, M. H., and Cagney, K. A. (2007). Cultural leverage: interventions using culture to narrow racial disparities in health care. Medical Care Research Review, 14(5): 243S-282S.

[8] Lu, M., Moritz, S., Lorenzetti, D., Sykes, L., Straus, S., and Quan, H. (2012). A systematic review of interventions to increase breast and cervical cancer screening uptake among Asian women. BMC Public Health, 12: 413.

[9] Smith, L. (2001). Evaluation of an educational intervention to increase cultural competence among registered nurses. Journal of Cultural Diversity, 14(2): 50-63.

[10] Bialocerkowski, A., Wells, C., and Grimmer-Somers, K. (2011). Teaching physiotherapy skills in culturally-diverse classes. BMC Medical Education, 11, 34-43.

[11] Kokko, R. (2011). Future nurses' cultural competencies: what are their learning experiences during exchange and studies abroad? A systematic literature review. Journal of Nursing Management, 19: 67382.

[12] Cushman, L., Delva, M., Franks, C., Jimenez-Bautista, A., Moon-Howard, J., Glover, J., and Begg, M. (2015). Cultural competency training for public health students: integrating self, social, and global awareness into a master of public health curriculum. American Journal of Public Health, 105: S132-S140.

[13] Dhaliwal, J., Crane, L., Valley, M., and Lowenstein, S. (2013). Student perspectives on the diversity climate at a U.S. medical school: the need for a broader definition of diversity. BMC Research Notes, 6: $154-61$.

[14] Elminowski, N. (2015). Developing and implementing a cultural awareness workshop for nurse practitioners. Journal of Cultural Diversity, 22(3): 105-13.

[15] Esterhuizen, P. and Kirkpatrick, M. (2015). Intercultural-global competencies for the 21st century and beyond. Journal of Continuing Education in Nursing, 46(5): 209-14.

[16] Hammerich, K. (2014). Commentary on a framework for multicultural education. Journal of the Canadian Chiropractic Association, 58(3): 280-5.

[17] Marin, R., Hawthorne, L., Morgan, M., and Ismail, M. (2012). Transcultural skills content in a dental curriculum: a comparative study. European Journal of Dental Education, 16: e33-e40. 
[18] Nuciforo, M. (2015). Minority applicants to physical therapist education programs. Physical Therapy, 95(1): 39-50.

[19] Oliveira, K., North, S., Beck, B., and Hopp, J. (2015). Promoting collaboration and cultural competence for physician assistant and physical therapist students: a cross-cultural decentralized interprofessional education model. Journal of Educational Evaluation for Health Professionals, 12: 20-4.

[20] Palombaro, K., Dole, R., and Black, J. (2015). Curricular integration and measurement of cultural competence development in a group of physical therapy students. Journal of the Scholarship of Teaching and Learning, 15(5): 82-96.

[21] Villarruel, A., Washington, D., Lecher, W., and Carver, N. (2015). A more diverse nursing workforce: greater diversity is good for the country's health. American Journal of Nursing, 115(5): 57-62.

[22] Wehbe-Alamah, H. and Fry, D. (2014). Creating a culturally sensitive and welcoming academic environment for diverse health care students: a model exemplified with Muslim physical therapist students. Journal of Physical Therapy Education, 28(1): 5-15.

[23] Yeowell, G. (2013). 'Isn't it all Whites?' Ethnic diversity and the physiotherapy profession. Physiotherapy, 99: 341-6.

[24] Zanetti, M., Dinh, A., Hunter, L., Godkin, M., and Ferguson, W. (2014). A longitudinal study of multicultural curriculum in medical education. International Journal of Medical Education, 5: 37-44.

\section{AUTHOR'S BIOGRAPHY}

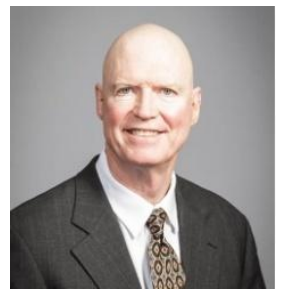

James M. McKivigan, Ph.D., DPT, DC, MA, MS, MBA, MPA, is an associate professor in the School of Physical Therapy at Touro University Nevada. He began working at Touro in 2009. Dr. McKivigan received his Ph.D. from Northcentral University in Arizona in 2018, Doctor of Physical Therapy at the University of Montana in 2013, and a Doctor of Chiropractic from Palmer College of Chiropractic in Davenport, Iowa in 2004. He is an active member of physical therapy, chiropractic, research, anatomical, and administrative associations. His research interests include interdisciplinary cooperation in the healthcare services, spinal manipulation, radiology, orthopedic rehabilitation, physical modalities, and electrotherapeutics

Citation: James McKivigan." Cultural Diversity in the Practice of Physical Therapy: A Review of the Literature" International Journal of Humanities Social Sciences and Education (IJHSSE), vol 8,no.1, 2021, pp.35-43. doi:https://doi.org/10.20431/2349-0381.0801004.

Copyright: (C) 2021 Authors. This is an open-access article distributed under the terms of the Creative Commons Attribution License, which permits unrestricted use, distribution, and reproduction in any medium, provided the original author and source are credited. 